\title{
Implante valvular aórtico transcatéter, experiencia de dos años en el Hospital Regional ISSSTE
}

\section{Transcatheter aortic valve implant, two-year experience at the ISSSTE Regional Hospital}

\author{
José F. Flores-Herrera ${ }^{1,2 *}$, Víctor M. Gudiño-Carballo ${ }^{2}$ y Miriam Navarrete-Sandoval ${ }^{2}$ \\ ${ }^{1}$ Facultad Mexicana de Medicina, Universidad La Salle México; ${ }^{2}$ Servicio de Terapia Intensiva, Hospital Regional General Ignacio Zaragoza, ISSSTE. \\ Ciudad de México, México
}

\begin{abstract}
Resumen
Introducción: La estenosis aórtica es la patología valvular más común, y la mortalidad a corto plazo es del $50 \%$ a los dos años en los pacientes no operados. El implante valvular aórtico transcatéter (TAVI) es el tratamiento de la estenosis aórtica de alto riesgo e inoperable y una opción terapéutica para los pacientes de riesgo intermedio. Material y métodos: Se realizó un estudio observacional retrolectivo de los pacientes sometidos a TAVI en un hospital de tercer nivel de la Ciudad de México. Resultados: Se incluyeron 14 pacientes, todos fueron electivos. El riesgo promedio calculado por el EuroSCORE Il fue de riesgo intermedio (con un promedio de 4.2\%) y por el STS (modelo de la Society of Thoracic Surgeons) fue del $5.6 \%$ ( \pm 6.5$)$. Se colocaron 11 prótesis Medtronic CoreValve ${ }^{\mathrm{TM}}$ y 3 Edwards SAPIEN XT ${ }^{\mathrm{TM}}$. Las complicaciones más frecuentes fueron: trastornos del ritmo en ocho casos, fuga paravalvular en tres casos y muerte en un caso. Conclusiones: EI TAVI es un procedimiento seguro, accesible y útil en población de riesgo intermedio.
\end{abstract}

Palabras clave: Estenosis de la válvula aórtica. Reemplazo de la válvula aórtica transcatéter. Enfermedades de las válvulas cardíacas.

\begin{abstract}
Introduction: Aortic stenosis is the most common valvular pathology and short-term mortality is (50\% at two years) in non-operated patients. Transcatheter aortic valve implantation (TAVI) is the treatment of high-risk and inoperable aortic stenosis (AS) and a therapeutic option for intermediate-risk patients. Material and methods: A retrolective observational study of patients undergoing TAVI was conducted at a third-level hospital in Mexico City. Results: Included 14 patients were all elective; had average risk calculated by EuroSCORE II was intermediate risk (averaging 4.2\%) and 5.6\% STS ( \pm 6.5 ) 11 Medtronic CoreValve ${ }^{\mathrm{TM}}$ and 3 Edwards SAPIEN XT ${ }^{\mathrm{TM}}$ prostheses were placed. Complications were reported as complications of rhythm eight disorders, paravalvular leakage three and death in one case. Conclusions: TAVI is a safe, accessible and useful procedure in intermediate risk populations.
\end{abstract}

Key words: Aortic valve stenosis. Transcatheter aortic valve replacement. Heart valve diseases.

Correspondencia:

*José F. Flores-Herrera

E-mail: dr_floresherrera@ hotmail.com
Fecha de recepción: 05-12-2019

Fecha de aceptación: 29-12-2019

DOI: $10.24875 /$ RHJM.20000043
Disponible en internet: 19-06-2020 Rev Hosp Jua Mex. 2020;87(2):65-69

www.revistahospitaljuarez.com 1405-9622/@ 2019 Sociedad Médico-Quirúrgica del Hospital Juárez de México, A.C. Publicado por Permanyer. Este es un artículo open access bajo la licencia CC BY-NC-ND (http://creativecommons.org/licenses/by-nc-nd/4.0/). 


\section{Introducción}

La estenosis aórtica (EA) es la valvulopatía más común y la tercera enfermedad cardiovascular más frecuente en el mundo occidental, después de la hipertensión arterial sistémica y la cardiopatía isquémica'. Su prevalencia aumenta con la edad: el $0.2 \%$ a los 50-59 años, el 1.3\% a los 60-69 años, el 3.9\% a los 70-79 años y el $9.8 \%$ a los 80-89 años. La principal etiología de la EA es degenerativa (81.9\%). En pacientes con regurgitación aórtica, la causa degenerativa también predominó $(50.3 \%)^{2}$. La aparición y progresión de los síntomas suele ser rápida, con una mortalidad alta a corto plazo (el $50 \%$ a los dos años) en los pacientes no operados 2 . La valvuloplastia con balón hoy se considera solo como una opción de emergencia y como puente hacia la cirugía o el implante valvular aórtico transcatéter (TAVI) $)^{3,4}$. El reemplazo valvular quirúrgico es aún el tratamiento de elección en pacientes con EA grave sin contraindicación para la cirugía. Sin embargo, en pacientes con edad avanzada y otras patologías asociadas, la mortalidad incrementa con la cirugía, por lo que muchos pacientes no son operados y su pronóstico vital es malo a corto plazo. EI TAVI es el tratamiento de la EA de alto riesgo e inoperable y una opción terapéutica para los pacientes de riesgo intermedio ${ }^{5}$. Las dos prótesis con más experiencia en el mundo son la prótesis balón expandible Sapien Edwards ${ }^{\mathrm{TM}}$ y la válvula autoexpandible CoreValve ${ }^{\mathrm{TM}}$ (Fig. 1) $)^{6,7}$. Las primeras series de centros únicos demostraron la viabilidad y eficiencia de esta modalidad de tratamiento. Posteriormente, estudios multicéntricos confirmaron su eficacia. En todo el mundo han determinado una rápida penetración de esta técnica ${ }^{8,9}$. Se han publicado dos trabajos con la válvula autoexpandible CoreValve ${ }^{\mathrm{TM}}$. El primero, en pacientes con EA severa, con un mayor riesgo quirúrgico, y el TAVI con una bioprótesis valvular aórtica CoreValve ${ }^{\mathrm{TM}}$ autoexpandible se asoció con una tasa de supervivencia significativamente mayor al año que el reemplazo quirúrgico de la válvula aórtica. El otro estudio concluyó que el TAVI con bioprótesis autoexpandible fue segura y efectiva en pacientes con EA grave sintomática en riesgo prohibitivo para el reemplazo quirúrgico ${ }^{10,11}$. En la actualidad se recomienda el TAVI como una posible intervención para pacientes considerados de alto riesgo quirúrgico o inoperables (clase IB). De manera crucial, tanto la European Society of Cardiology (ESC) como la American Heart Association/American College of Cardiology (AHA/ACC) recomiendan que todos los casos potenciales de TAVI deben ser discutidos por un equipo multidisciplinario (Heart Team). Ambas pautas

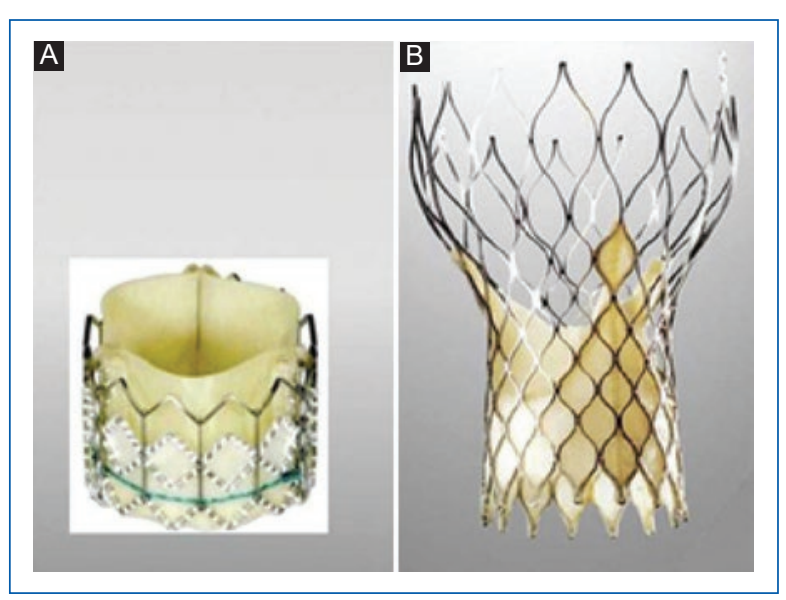

Figura 1. A: Válvula Edwards-Sapien. B: Medtronic CoreValve.

también recomiendan utilizar escalas de riesgo quirúrgico, incluido el EuroSCORE logístico o el STS (modelo de la Society of Thoracic Surgeons) para identificar los pacientes con alto riesgo ${ }^{12,13}$. El objetivo del presente trabajo es presentar la experiencia durante este periodo de dos años en el Hospital Regional General Ignacio Zaragoza del Instituto de la Seguridad y Servicios Sociales de los Trabajadores del Estado y compararla con la literatura.

\section{Material y métodos}

Se incluyeron todos los pacientes con EA grave que se trataron mediante TAVI en la Unidad de Terapia Intensiva en el periodo de enero de 2017 a febrero de 2019 (muestra consecutiva a conveniencia del investigador). Como protocolo diagnóstico para la selección de los pacientes, se realizaron exámenes generales, ecocardiografía transtorácica y tomografía computada multicorte. Ambos métodos se utilizaron para evaluar las dimensiones de la aorta y de la válvula aórtica, así como su morfología y el grado y distribución de las calcificaciones. Todos los pacientes fueron sometidos a coronariografía y aortografía previo al procedimiento (Tabla 1). El procedimiento se realizó de acuerdo con la técnica y protocolos descritos en la literatura.

\section{Descripción de los dispositivos}

La prótesis Medtronic CoreValve ${ }^{\mathrm{TM}}$ es una prótesis trivalvular biológica de pericardio porcino, montada y suturada en un marco de stent autoexpandible de nitinol. El sistema de liberación tiene un tamaño de 18 French. La válvula Edwards SAPIEN XT ${ }^{\mathrm{TM}}$ es un stent 
Tabla 1. Características de los pacientes

\begin{tabular}{|l|c|}
\hline Característica & (n = 14) \\
\hline Edad (años) & $75.8 \pm 5.84$ \\
Sexo & \\
Femenino & 57 \\
Masculino & 43 \\
IMC (kg/m²) & $22.79 \pm 2.81$ \\
\hline Historia médica & \\
Cirugía bypass previa (\%) & 0 \\
\hline Angioplastia previa (\%) & $6(42.8)$ \\
\hline Cirugía valvular previa (\%) & 0 \\
\hline Valvuloplastia con balón previa (\%) & $1(7.1)$ \\
\hline Accidente vascular previo (\%) & 0 \\
\hline Diabetes mellitus (\%) & $6(42.8)$ \\
EPOC (\%) & $1(7.1)$ \\
\hline Enfermedad vascular periférica (\%) & 0 \\
Insuficiencia renal (\%) & $2(14.2)$ \\
Marcapasos previo (\%) & $1(7.1)$ \\
\hline Presentación clínica & \\
Angina (\%) & $7(50)$ \\
Síncope (\%) & $1(7.1)$ \\
NYHA II (\%) & $8(57.1)$ \\
NYHA III (\%) & $6(42.8)$ \\
NYHA IV (\%) & 0 \\
EuroSCORE logístico (\%) & 4.2 \\
STS (\%) & 5.6 \\
\hline
\end{tabular}

de cromo cobalto, y el sistema de liberación es compatible con un introductor de 18-20 French.

\section{Características de la población}

La fracción de eyección del ventrículo izquierdo (FEVI) promedio fue de $47 \pm 12.89 \%$, una paciente (7.14\%) presentaba una FEVI severamente deprimida, con valor menor a $30 \%$. El gradiente transvalvular máximo promedio fue de $49 \mathrm{mmHg}$ y la media de 44.15 \pm 3.93 , con velocidades máximas promedio de $5.3 \pm$ $0.55 \mathrm{~m} / \mathrm{s}$. La edad promedio de la población fue de 75 \pm 6.1 años, el $57 \%$ eran mujeres y el $43 \%$ hombres y el EuroSCORE II fue de 4.2, el STS promedio de 5.6\%. Las comorbilidades fueron: hipertensión arterial sistémica $(71.42 \%)$, seis pacientes $(42.8 \%)$ con diabetes mellitus y dos pacientes (14.2\%) con enfermedad renal crónica. La insuficiencia cardíaca clase $\geq$ III de la NYHA (New York Heart Association) estaba presente en el $35 \%$ de los pacientes. El EuroSCORE II fue de bajo riesgo (con un promedio de $4.2 \%$ ) y el STS fue del $5.6 \%( \pm 6.5)$. La mayoría de los pacientes tenían otras comorbilidades, que no se valoran en el EuroSCORE o el STS, que los hacía de riesgo quirúrgico intermedio y elevado. El $100 \%$ de los pacientes tenía una válvula aórtica tricúspide y el $21.4 \%$ de forma
Tabla 2. Hemodinamia y angiografía

\begin{tabular}{|l|c|}
\hline $\begin{array}{l}\text { Fracción de eyección del ventrículo } \\
\text { izquierdo (FEVI) }\end{array}$ & $\mathbf{n}(\mathbf{1 4})$ \\
\hline $\begin{array}{l}\text { Fracción de eyección promedio (\%) } \\
\text { Reducción severa (<30\%) }\end{array}$ & $47 \pm 12.8$ \\
\hline $\begin{array}{l}\text { Características de la válvula } \\
\text { Área valvular cm }\end{array}$ & $1(7.1)$ \\
$\quad$ Gradiente medio (mmHg) & \\
Válvula bicúspide (\%) & $0.5 \pm 0.14$ \\
$\quad$ Calcificación severa (\%) & $44.15 \pm 3.9$ \\
$\quad \begin{array}{l}\text { Diámetro del anillo (mm) } \\
\text { Insuficiencia aórtica concomitante grado I }\end{array}$ & 0 \\
\hline II (\%) & $10(71.4)$ \\
\hline $\begin{array}{l}\text { Otros hallazgos } \\
\text { Insuficiencia mitral concomitante grado I }\end{array}$ & $3(21.4)$ \\
\hline $\begin{array}{l}\text { Fibrilación auricular (\%) } \\
\text { Marcapasos previo (\%) }\end{array}$ & $1(7.1)$ \\
\hline
\end{tabular}

concomitante con insuficiencia valvular aórtica grado I a II. Se observó insuficiencia mitral grado I en el $7.14 \%$. Había dos pacientes que se encontraban en fibrilación auricular previo al procedimiento (Tabla 2). Las principales indicaciones de TAVI fueron riesgo quirúrgico medio, elevado o inoperables por sus múltiples comorbilidades. Todos los casos fueron discutidos en la sesión médico-quirúrgica integrada por los Servicios de Cardiología, Cirugía Cardiotorácica y Terapia Intensiva de nuestra unidad hospitalaria.

\section{Resultados}

Todos los pacientes fueron abordados por acceso femoral quirúrgico mínimo. No se observaron complicaciones vasculares durante el procedimiento. EI TAVI fue principalmente un procedimiento electivo (92.85\%), solo en una paciente $(7.14 \%)$ se realizó de emergencia. Se realizaron tres $(21.42 \%)$ bajo anestesia general y $11(78.57 \%)$ con sedoanalgesia. La duración promedio de la intervención fue de $146.6 \pm 11.4 \mathrm{~min}$. Se utilizó en promedio $140 \pm 34.22 \mathrm{ml}$ de medio de contraste, Ultravist ${ }^{\circledR}$. El gradiente transaórtico residual posprocedimiento fue de $6.7 \pm 2.4 \mathrm{mmHg}$. Se utilizaron 11 prótesis CoreValve Evolut $\mathrm{R}^{\mathrm{TM}}$ (78.57\% de los pacientes) y tres prótesis Edwards-SAPIEN XT'TM $(21.42 \%$ de los pacientes). La insuficiencia aórtica residual al final del procedimiento se observó en el $92.85 \%$ de los procedimientos, sin embargo, no se observó insuficiencia aórtica severa ( $\geq$ grado III). De los ocho $(57 \%)$ pacientes que presentaron trastornos del ritmo y conducción, a uno (12.5\%) se le implantó un marcapasos definitivo en el periodo de seguimiento del protocolo, fue por 
bloqueo auriculoventricular (AV) de tercer grado. De los tres $(21 \%)$ pacientes que presentaron fugas paravalvulares, el $100 \%$ fueron leves. No presentaron complicaciones hemorrágicas, renales, isquémicas 0 neurológicas. La mortalidad fue del 7\% (un paciente). Los días de estancia en Unidad de Terapia Intensiva fue de mínimo dos días y máximo cinco días, con una desviación estándar de \pm 0.96 .

\section{Discusión}

El TAVI adquiere una gran relevancia en el tratamiento de la EA grave sintomática en pacientes con riesgo quirúrgico intermedio 0 inoperables ${ }^{14,15}$. Esta serie de 14 pacientes demuestra la experiencia de todo el equipo multidisciplinario. Para establecer la indicación de TAVI, es importante la estimación precisa del riesgo quirúrgico. Se utilizaron calculadoras de riesgo, específicamente el modelo de la STS y el EuroSCORE. Estos formatos de evaluación no incluyen todas las variables que pueden influir en la morbimortalidad de un paciente ${ }^{16}$. En nuestra serie, el promedio del EuroSCORE fue del $4.2 \%( \pm 7.89)$ y el del STS de 5.6 $( \pm 6.56)$. Ambas escalas predicen un riesgo bajo o intermedio de mortalidad. Existen dos estudios en pacientes con EA grave y riesgo intermedio en los que se observan resultados similares en la mortalidad global a los 30 días y un año después del TAVI o reemplazo quirúrgico ${ }^{17,18}$. Estudios aleatorizados en curso, han sido diseñados para proporcionar una evidencia más sustentable en pacientes de riesgo intermedio (PARTNER II cohorte A con la válvula Edwards SAPIEN $X T^{T M}$ en EE. UU., y el estudio SURTAVI con la válvula CoreValve $^{\mathrm{TM}}$ de Medtronic). Por lo tanto, todavía no existe la suficiente evidencia científica que justifique el TAVI en pacientes de riesgo intermedio. En nuestro hospital se evaluaron otras comorbilidades no valoradas en las escalas de medición específicas. Un metaanálisis de 2012 utilizó los primeros criterios VARC (Valve Academic Research Consortium) para unificar los resultados: describieron todas las causas de mortalidad hasta los 30 días de TAVI, de un 1.7-14.3\% ${ }^{19}$. La mortalidad en nuestra serie, a los 30 días, fue del $7.14 \%$. Aunque es una serie con pocos pacientes, los resultados están dentro de lo que se menciona en la literatura. Una de las complicaciones más catastróficas son los eventos cerebrovasculares, la tasa dentro de los 30 primeros días es de alrededor del $3.6 \%$, según lo reportado en el estudio PARTNER $A^{20}$, hasta el $11.2 \%$ reportado en el PARTNER $B^{21}$. En nuestra población no se presentaron complicaciones mayores (infarto cerebral, infarto agudo al miocardio), esto es menor a lo reportado en la literatura. Las imágenes no invasivas tienen un papel crucial en la selección de pacientes y para la elección del tamaño de la válvula con el fin de asegurar el despliegue preciso de la prótesis, minimizar las complicaciones trans y posprocedimiento. Los estudios diagnósticos previos, como tomografías, aportaron datos sobre diámetros, grado de calcificación de los accesos vasculares, cuantificación, distribución del calcio valvular, morfología de la aorta ascendente y mediciones del anillo. La complicación más frecuente es la necesidad de implantar un marcapasos definitivo por el bloqueo AV de tercer grado intermitente o persistente. Cuando se utilizó CoreValve llegó a hasta el $42 \%^{22}$. En nuestra serie se implantó marcapasos definitivo en el $7.5 \%$ de los pacientes, que está dentro de los rangos reportados por otros centros. En nuestra población se instaló en todos los pacientes marcapasos temporal ante bloqueos transitorios postimplante para evitar mortalidad luego de un procedimiento endovascular exitoso. La insuficiencia aórtica es una complicación presente hasta en el $72.4 \%$ de los pacientes ${ }^{22}$. En nuestra serie fue del $78.5 \%$ (grado I o II). La evaluación de la insuficiencia residual se realizó con ecografía transtorácica y aortografía. Los datos disponibles de ambos tipos de válvulas son similares en este sentido, alcanzando tasas aproximadas del $70 \%$, con insuficiencia leve a moderada ${ }^{23,24}$.

\section{Limitaciones}

Se trató de un estudio retrolectivo, observacional, de una cohorte pequeña de pacientes en un solo centro, sin embargo, el número de casos registrados es parecido al total de otras series similares. Es un procedimiento de inclusión reciente en el hospital sede del estudio, por lo que el volumen de pacientes aún no llega al pico para los centros más experimentados.

\section{Conclusiones}

En la actualidad, el número de pacientes a los que se realizó TAVI en la población de mayor riesgo evidencia su eficiencia y seguridad. En un futuro las complicaciones serán mínimas y se podrán extender sus indicaciones ${ }^{25}$, lo que llevará a una mayor supervivencia de nuestra población. Esta opción de tratamiento debe ser accesible para los pacientes que lo necesiten y estar disponible en las instituciones de referencia de 
enfermedades cardiovasculares que tengan los recursos materiales y humanos.

\section{Conflicto de intereses}

Los autores declaran no tener conflicto de intereses alguno.

\section{Financiamiento}

Los autores no recibieron patrocinio para llevar a cabo este artículo.

\section{Responsabilidades éticas}

Protección de personas y animales. Los autores declaran que para esta investigación no se han realizado experimentos en seres humanos ni en animales.

Confidencialidad de los datos. Los autores declaran que han seguido los protocolos de su centro de trabajo sobre la publicación de datos de pacientes.

Derecho a la privacidad y consentimiento informado. Los autores declaran que en este artículo no aparecen datos de pacientes.

\section{Bibliografía}

1. Lung B, Baron G, Butchart EG, Delahaye F, Gohlke-Bärwolf C Levang OW, et al. A prospective survey of patients with valvular heart disease in Europe: The Euro Heart Survey on Valvular Heart Disease. Eur Heart J. 2003;24:1231-43.

2. Morís C, Pascual I, Avanzas P. ¿Será el TAVI el tratamiento de elección para la estenosis aórtica? Rev Esp Cardiol. 2016:(69):1131-4.

3. Cribier A, Savin T, Saoudi N, Rocha F, Letac B. Percutaneous transluminal valvuloplasty of acquired aortic stenosis in elderly patients: An alternative to valve replacement? Lancet.1986:1:63-7.

4. Nkomo VT, Gardin JM, Skelton TN, Gottdiener JS, Scott CG Enriquez-Sarano, M. Acute and 30-dayfollow-up results in 674 patients from the Balloon Valvuloplasty registry. Lancet. 2006;368:1005-11.

5. Martín P, Hernández R, Barrios R, Gutiérrez H, Ojeda J, Páez L, et al. Implantación de válvula aórtica por cateterismo cardiaco. TAVI. Rev Sanid Milit Mex. 2014;68(2):108-21

6. Caorsi C, Baeza C, Morís C, Oyonarte M, Venegas JC, Yovanovich J, et al. Implante valvular aórtico transarterial: experiencia en Chile. Rev Méd Chile. 2013;141(1):7-14

7. Martin-Hernandez P, Gutierrez-Leonard H, Ojeda-Delgado JL, Fagoaga-Valdivia J, Barrios-Nanni R, et al. Experiencia de la implantación de válvulas aórticas transcatéter en el Hospital Central Militar. Arch Cardiol Mex. 2015;85(4):296-306.

8. Thomas M. 30-day results of the Source registry: The European registry of transcatheter aortic valve implantation using the Edwards Sapien valve.
Conferencia EuroPCR. Mayo 2009. Disponible en: http://www.pcronline. com/EuroPCR/EuroPCR2009/Transcatheter aortic-valve-implantation registries.

9. Lardizabal JA, Macon CJ, O'Neil BP, et al. Clinical outcomes whit on label and off label and of label use of the transcatheter heart valve in the United States. Catheter aortic valve replacement with a self expanding prosthesis. N Engl J Med. 2014;370:1790-8.

10. Adams DH, Popma JJ, Reardon MJ, et al. Transcatether aortic valve replacement with a self expanding prosthesis. N Engl J Med. 2014;370:1790-8.

11. Popma JJ, Adams DH, Reardon MJ, et al. Transcatether aortic valve replacement using a self expanding bioprosthesis in patients with severe aortic stenosis at extreme risk for surgery. J Am Coll Cardiol. 2014;631972-81.

12. Nishimura RA, Otto $C M$, Bonow RO, et al. AHA/ACC guideline for the management of patients with valvular heart disease: A report of the American College of Cardiology/American Heart Association Task Force on Practice Guidelines. J Am Coll Cardiol. 2014;63:e57-185.

13. Van Mieghem NM, Head SJ, Van der Boon RM, Piazza N, de Jaegere PP Carrel T, et al. The SURTAVI model: Proposal for pragmatic risk stratification for patients with severe aortic stenosis. Euro Intervention. 2012;8:258-66.

14. Martin-Hernández $\mathrm{P}$, Gutiérrez-Leonard H, Ojeda-Delgado JL, Páez Lizárraga LM, Alcocer-Macías JJ, Fagoaga-Valdiviam J, et al. Implante de válvula aortica transcatéter en una válvula aórtica bicúspide en el Hospital Central Militar México. Rev Sanid Milit Mex. 2014;68:52-4.

15. Eid-Lidt G, Gaspar J, Arias A, Ramírez-Marroquín S, Damas F, Herrera V, et al. Start-up of the program of transcatheter aortic valve implantation using a balloon expandable Edwards Sapien XT transcatheter heart valve. Description of the first case in Mexico. Arch Cardiol Mex. 2014:84:133-5.

16. Ben-Dor I, Gaglia MA Jr, Barbash IM, Maluenda G, Hauville C, Gonzalez MA, et al. Comparison between Society of Thoracic Surgeons score and logistic EuroSCORE for predicting mortality in patients referred for transcatheter aortic valve implantation. Cardiovasc Revasc Med. 2011;12:345-9.

17. Piazza N, Kalesan B, van Mieghem N, Head S, Wenaweser P, Carrel TP, et al. A 3-center comparison of 1-year mortality outcomes between transcatheter aortic valve implantation and surgical aortic valve replacement on the basis of propensity score matching among intermediate risk surgical patients. JACC Cardiovasc Interv. 2013;6:443-51.

18. D'Errigo P, Barbanti M, Ranucci M, Onorati F, Covello RD, Rosato S, et al.; OBSERVANT Research Group. Transcatheter aortic valve implantation versus surgical aortic valve replacement for severe aortic stenosis: results from an intermediate risk propensity-matched population of the talian OBSERVANT study. Int J Cardiol. 2013;167:1945-52.

19. Genereux P, Head SJ, van Mieghem NM, Kodali S, Kirtane AJ, Xu K, et al. Clinical outcomes after transcatheter aortic valve replacement using valve academic research consortium definitions: A weighted meta analysis of 3,519 patients from 16 studies. J Am Coll Cardiol. 2012;59:2317-26.

20. Smith CR, Leon MB, Mack MJ, Miller DC, Moses JW, Svensson LG, et al. PARTNER Trial Investigators. Transcatheter versus surgical aortic-valve replacement in high risk patients. N Engl J Med. 2011;364:2187-98.

21. Leon MB, Smith CR, Mack M, Miller DC, Moses JW, Svensson LG, et al. Transcatheter aortic-valve implantation for aortic stenosis in patients who cannot undergo surgery. N Engl J Med. 2010;363:1597-607.

22. Kahn R, Gerckens U, Grube E, Linke A, Sievert H, Eggebrecht H, et al. Transcatheter aortic valve implantation: first results from a multi centre real world registry. Eur Heart J. 2011;32:198-204.

23. Grube E, Buellesfeld L, Mueller R, Sauren B, Zickmann B, Nair D, et al. Progress and current status of percutaneous aortic valve replacement: results of three devicgenerations of the CoreValve Revalving system. Circ Cardiovasc Interv. 2008:1:167-75.

24. Webb JG, Altwegg L, Boone RH, Cheung A, Ye J, Lichtenstein S, et al. Transcatheter aortic valve implantation: impact on clinical and valve-related outcomes. Circulation 2009;119:3009-16.

25. Piazza N, Grube E, Gerckens U, den Heyer P, Linke A, Luha O, et al. Prodedural and 30-day outcomes following transcatheter aortic valve implantation using the third generation $(18 \mathrm{~F})$ CoreValve Revalving sysem: results from the multicentre, expanded evaluation registry 1-year following CE mark approval. Eurolntervention. 2008;4:242-9. 